\title{
GENETIC ALGORITHM PARAMETERS OPTIMIZATION FOR ELECTRICAL DISCHARGE MACHINING USING DESIGN OF EXPERIMENTS
}

\author{
Amit Kumar(Corresponding author $)^{1} \&$ Ashwani Kumar Dhingra ${ }^{2}$
}

\begin{abstract}
Present work considers the Genetic Algorithm (GA) parameters optimization for the Material Removal Rate (MRR) and Tool Wear Rate (TWR) for the Electric Discharge Machining using the Design of Experiments. Efficiency and effectiveness of genetic algorithm can be achieved by optimization of its different parameters such as crossover, mutation, crossover probability, selection function etc. Proper selection of genetic algorithm parameters plays an important role in the optimization. The central composite design (CCD) of Response Surface Methodology (RSM) has been used for the optimization. The experiments have been performed with 5 factors. The regression equation for MRR and TWR derived by Periyakgounder et. al. [10] has been used as an objective function in the genetic algorithm and the parameters of GA have been optimized using Response Surface Methodology (RSM). The experiments have been performed with different levels of GA parameters and significant parameters of the genetic algorithm for the optimised MRR and TWR has been reported.

Keywords: Genetic Algorithm, Material Removal Rate, Tool Wear Rate, Electric Discharge Machining
\end{abstract}

\section{INTRODUCTION}

Electric Discharge Machining (EDM) is a non-traditional electro-thermal machining process, in which material removal occurs due to thermal energy produced by the spark. Electric discharge machining (EDM) is mainly used for machining of materials that are difficult to machines like ceramics, maraging steels, cast-alloys and titanium having an application in defense and aerospace industries. EDM is also used to handle irregular geometries in small lots. Work material should be electrically conductive to be machined by EDM. The working current, working voltage, oil pressure, polarity, pulse current , pulse on time, pulse of time and electrode gap are the electrical parameters. The non-electrical parameters like work piece, tool material and dielectric fluid pressure mainly influence the performance of this process. The EDM output parameters like MRR, TWR and SR are affected significantly by these electrical and non electrical parameters. It is very complex to determine the optimum machining parameters for EDM. The output responses MRR and TWR are contradictory in nature. The productivity is reflected by MRR and the accuracy of the product is reflected by the tool wear. Therefore, the effects of different input parameters like current, pulse on time and pulse off time on MRR and TWR with EDM oil as a dielectric has been considered in the present work.

\section{LITERATURE REVIEW}

Tzeng et. al.[1] is focused in the optimization of process parameter by using back-propagation neural network (BPNN), a genetic algorithm (GA), and response surface methodology (RSM) . Yadav et.al. [2] focused ithe maximizing material removal rate by using artificial neural network (ANN) and grey relational analysis (GRA) for the process optimization with the parameters such as pulse current, pulse on- time and duty factor for the optimisation of MRR and SR. The work shows that process parameters namely pulse on time has a very significant effect on MRR . Radhika et. al. [3] worked on Electric Discharge Machining of aluminium hybrid Metal Matrix Composite. Multi-objective optimization of process parameters has been performed by ANN and GA. Qing et. al. [4] obtained ther parameters optimization model using ANN and GA. The relationship between MRR and input parameter represented by ANN model adapts Levenberg-Marquardt algorithm has been derived for the optimization of results. The MRR has been maximized by the optimized machining parameters. Using pulse on and proper pulse off time setting with the same current, the MRR is being improved. Balasubramanian et. al. [5] optimized the machining parameters in EDM with the workpiece material of Carbon Steel Bar En8 and D3 steel material have been used. The process parameters pulse on time, pulse of time, peak current, die electric pressure and tool diameter have been consider for the analysis. The output responses are Tool Wear Rate (TWR), Material Removal Rate (MRR), and Surface Roughness (SR). Tiwari[6] used the titanium alloy Ti 6-4 as working piece material for EDM machining. Surface hardness

\footnotetext{
${ }^{1}$ Department of Mechanical Engineering, University Institute of Engineering and Technology, Maharishi Dayanand University, Rohtak, Haryana (124001) India

${ }^{2}$ Department of Mechanical Engineering, University Institute of Engineering and Technology, Maharishi Dayanand University, Rohtak, Haryana (124001) India
} 
and Material removal rate have been optimized by the genetic algorithm. The current and pulse on time have been directly proportional to MRR with pulse off time being inversely proportional. Ahmad et. al. [7] describe that the MRR is the important performance attributes for the EDM process. MRR depends on the size of machining surface. The input parameters considered are Peak Current (Ip), Pulse Time On (Ton), Duty Cycle (TAU) and Voltage Gap (V). Design of experiments based on L18 Orthogonal Array (OA) has been considered. Optimal parameters for MRR on Titanium grade-2 with copper electrode found to be 12 -amp peak current, $100-\mu$ sec pulse time on, $15 \%$ duty cycle and 60 volt voltage gap. Harmanpreet et. al. [8] conducted experiments using Design of experiment with mixed level L18 orthogonal array. All the 18 experiments were performed on INCOLOY-800 with copper material as an electrode. Two types of dielectric fluid have been considered as kerosene and EDM oil for the multi objective-optimization Genetic Algorithm (GA) . Laxman et al.[9] worked on hard metals using complex shapes Electro Discharge Machining. Regression analysis has been used for the correlation between various process parameters. The Metal removal rate, electrode wearing rate and surface roughness considered as responses and the working current, pulse on time, pulse off time, tool lift time, working voltage as the input parameters. Increase in pulse off time and pulse on time effects the MRR, TWR and SR

\section{PROBLEM STATEMENT}

Poor machining rate or performance may be caused by an improper combination of process parameters in EDM. Through extensive machining processes which leads to lesser material removal rate is the basic characteristic related to MRR; or in other words, the reduced material removal rate is an attribute of MRR. The fertility of this effort makes it unsuitable for the production. The decrease in the accuracy of the product caused by the effects of the TWR characteristic is the second problem. Periyakgounder et al.[10] obtained better MRR and TWR simultaneously for the Electric Discharge Machining (EDM) using regression equation:

With help of this regression equation the fitness function is created in GA.

$\operatorname{MRR}=0.6688+0.07685 \mathrm{x}_{1}-0.15883 \mathrm{x}_{3}-0.31122 \mathrm{x}_{1}+\left(0.011663 \mathrm{x}_{1} \mathrm{x}_{2}\right)+\left(0.028113 \mathrm{x}_{1} \mathrm{x}_{3}\right)+\left(0.028438 \mathrm{x}_{2} \mathrm{x}_{3}\right)$

TWR $=-0.38495142+0.05307984 \mathrm{x}_{1}+0.0563238 \mathrm{x}_{1}-0.00393730 \mathrm{x}_{3}-\left(0.00357793 \mathrm{x}_{1} \mathrm{x}_{2}\right)+\left(0.0004195 \mathrm{x}_{1} \mathrm{x}_{3}\right)-$ $\left(0.00178412 \mathrm{x}_{2} \mathrm{x}_{3}\right)$,

Objective $1=1 /$ MRR,

Objective $2=$ TWR,

subject to: $6 \leq \mathrm{x} 1 \geq 12$,

$3 \leq x 2 \geq 9$,

$3 \leq \mathrm{x} 3 \geq 9$.

Where $\mathrm{x} 1=$ current, $\mathrm{x} 2=$ pulse on time, $\mathrm{x} 3=$ pulse off time .

\section{RESULT AND DISCUSSIONS}

The optimizations of different parameters of GA for MRR and TWR have been considered in the present work. population size, crossover, mutation and many other such parameters of genetic algorithm come under the purview of the study. In order to improve the performance of GA, a number of methods have been developed. The optimal parameters selection for the solution of the particular problem is one of the methods for improving the performance of Genetic Algorithms. For the optimization of the MRR and TWR, a Central Composite Design (CCD) of Response surface methodology (RSM) with Design Expert 6.0 has been considered with different genetic algorithm parameters. Table I shows the range and levels of the GA parameters considered. The design matrix is the result of the combination of various variables which requires 156 experiments. Running five times for taking final average, the genetic algorithm is related to the category of approximate algorithms. Therefore, total of $156 \times 5=780$ results have been obtained for the optimization of GA parameters for which the design summary is shown in table 2 in which two factors (Population Size(A) and Crossover (B) are numeric and the other factors Creation Function (C), Mutation (D) and Crossover function (E) are categorical.

Table 1: Genetic Algorithm Parameters with Range and Levels

\begin{tabular}{|l|l|l|}
\hline S.No. & Parameters (factors) & Range \\
\hline 1 & Population Size (A) & $20-80$ (5 levels) \\
\hline 2 & Cross over (B) & $0.50-0.90$ (5 levels) \\
\hline 3 & Creation function(C) & Level 1 (unifrom ), level 2(Feasible population) \\
\hline 4 & Mutation (D) & Level 1(uniform), level 2(adaptive feasible) \\
\hline 5 & Crossover function (E) & $\begin{array}{l}\text { level 1(scattered), level 2(single point) } \\
\text { Level 3(two point) }\end{array}$ \\
\hline
\end{tabular}

Table 2: Design Summary

\begin{tabular}{|l|l|l|l|l|l|l|l|}
\hline Response & Name & Units & Obs & Minimum & Maximum & Trans & Model \\
\hline Y1 & MRR & 0 & 156 & 1.49 & 2.40 & None & Quadratic \\
\hline Y2 & TWR & 0 & 156 & 0.67 & 0.77 & None & Quadratic \\
\hline
\end{tabular}




\begin{tabular}{|l|l|l|l|l|l|l|l|}
\hline Factor & Name & Units & Type & Low Actual & High Actual & Low Coded & High Coded \\
\hline A & Population size & 0 & Numeric & 20.00 & 70.00 & -1.000 & 1.000 \\
\hline B & Crossover fraction & 0 & Numeric & 0.50 & 0.90 & -1.000 & 1.000 \\
\hline C & Creation function & 0 & Categorical & Unifrom & Feasible population & 0 & Levels: 2 \\
\hline D & Mutation & 0 & Categorical & Unifrom & Adaptive feasible & 0 & Levels: 2 \\
\hline E & Crossover function & 0 & Categorical & Scattered & Two point & 0 & Levels: 3 \\
\hline
\end{tabular}

Table 3: Different Parameters Combination of GA using RSM

\begin{tabular}{|c|c|c|c|c|c|c|c|c|}
\hline Std & Run & $\begin{array}{l}\text { Factor1 } \\
\text { A:Population } \\
\text { size }\end{array}$ & $\begin{array}{l}\text { Factor2 } \\
\text { B:Crossover } \\
\text { fraction }\end{array}$ & $\begin{array}{c}\text { Factor3 } \\
\text { C:Creation } \\
\text { function }\end{array}$ & $\begin{array}{l}\text { Factor4 } \\
\text { D:Mutation } \\
\text { function }\end{array}$ & $\begin{array}{l}\text { Factor5 } \\
\text { E:Crossover } \\
\text { function }\end{array}$ & $\begin{array}{l}\text { Response1 } \\
\text { MRR }\end{array}$ & $\begin{array}{r}\text { Response2 } \\
\text { TWR }\end{array}$ \\
\hline 9 & 1 & 45 & 0.7 & Unifrom & Unifrom & Scattered & 2.168 & 0.701 \\
\hline 89 & 2 & 45 & 0.7 & Unifrom & Adaptive feasible & Single point & 2.28 & 0.691 \\
\hline 14 & 3 & 20 & 0.5 & Feasible population & Unifrom & Scattered & 2.169 & 0.706 \\
\hline 121 & 4 & 70 & 0.9 & Feasible population & Unifrom & Two point & 2.147 & 0.703 \\
\hline 118 & 5 & 20 & 0.5 & Feasible population & Unifrom & Two point & 1.648 & 0.732 \\
\hline 131 & 6 & 20 & 0.5 & Unifrom & Adaptive feasible & Two point & 2.185 & 0.698 \\
\hline 32 & 8 & 80 & 0.7 & Unifrom & Adaptive feasible & Scattered & 2.273 & 0.691 \\
\hline 17 & 9 & 70 & 0.9 & Feasible population & Unifrom & Scattered & 2.11 & 0.71 \\
\hline 111 & 10 & 45 & 0.4 & Unifrom & Unifrom & Two point & 2.24 & 0.696 \\
\hline 103 & 11 & 45 & 0.7 & Feasible population & Adaptive feasible & Single point & 2.27 & 0.69 \\
\hline 78 & 12 & 45 & 0.7 & Feasible population & Unifrom & Single point & 2.07 & 0.715 \\
\hline 28 & 13 & 70 & 0.5 & Unifrom & Adaptive feasible & Scattered & 2.284 & 0.691 \\
\hline 38 & 14 & 45 & 0.7 & Unifrom & Adaptive feasible & Scattered & 2.26 & 0.692 \\
\hline 83 & 15 & 10 & 0.7 & Unifrom & Adaptive feasible & Single point & 1.587 & 0.742 \\
\hline 115 & 16 & 45 & 0.7 & Unifrom & Unifrom & Two point & 2.195 & 0.698 \\
\hline 47 & 17 & 45 & 1.0 & Feasible population & Adaptive feasible & Scattered & 2.283 & 0.691 \\
\hline 140 & 18 & 45 & 0.7 & Unifrom & Adaptive feasible & Two point & 2.196 & 0.704 \\
\hline 93 & 20 & 70 & 0.5 & Feasible population & Adaptive feasible & Single point & 2.285 & 0.7 \\
\hline 13 & 21 & 45 & 0.7 & Unifrom & Unifrom & Scattered & 2.212 & 0.699 \\
\hline 130 & 22 & 45 & 0.7 & Feasible population & Unifrom & Two point & 1.846 & 0.73 \\
\hline 98 & 23 & 45 & 0.4 & Feasible population & Adaptive feasible & Single point & 2.281 & 0.67 \\
\hline 88 & 24 & 45 & 0.7 & Unifrom & Adaptive feasible & Single point & 2.281 & 0.691 \\
\hline 63 & 25 & 45 & 0.7 & Unifrom & Unifrom & Single point & 2.199 & 0.698 \\
\hline 44 & 26 & 10 & 0.7 & Feasible population & Adaptive feasible & Scattered & 2.08 & 0.715 \\
\hline 59 & 27 & 45 & 0.4 & Unifrom & Unifrom & Single point & 2.284 & 0.71 \\
\hline 27 & 28 & 20 & 0.5 & Unifrom & Adaptive feasible & Scattered & 2.24 & 0.691 \\
\hline 30 & 29 & 70 & 0.9 & Unifrom & Adaptive feasible & Scattered & 2.246 & 0.694 \\
\hline 108 & 30 & 70 & 0.9 & Unifrom & Unifrom & Two point & 2.055 & 0.713 \\
\hline 114 & 31 & 45 & 0.7 & Unifrom & Unifrom & Two point & 2.172 & 0.7075 \\
\hline 51 & 32 & 45 & 0.7 & Feasible population & Adaptive feasible & Scattered & 2.279 & 0.6915 \\
\hline 134 & 33 & 70 & 0.9 & Unifrom & Adaptive feasible & Two point & 2.275 & 0.694 \\
\hline 62 & 34 & 45 & 0.7 & Unifrom & Unifrom & Single point & 1.8642 & 0.705 \\
\hline 90 & 35 & 45 & 0.7 & Unifrom & Adaptive feasible & Single point & 2.279 & 0.691 \\
\hline 86 & 36 & 45 & 1.0 & Unifrom & Adaptive feasible & Single point & 2.124 & 0.7075 \\
\hline 84 & 37 & 80 & 0.7 & Unifrom & Adaptive feasible & Single point & 2.218 & 0.6965 \\
\hline 58 & 38 & 80 & 0.7 & Unifrom & Unifrom & Single point & 2.2285 & 0.696 \\
\hline 65 & 39 & 45 & 0.7 & Unifrom & Unifrom & Single point & 2.089 & 0.704 \\
\hline 91 & 40 & 45 & 0.7 & Unifrom & Adaptive feasible & Single point & 2.28 & 0.691 \\
\hline 73 & 44 & 45 & 1.0 & Feasible population & Unifrom & Single point & 2.128 & 0.709 \\
\hline 2 & 45 & 70 & 0.5 & Unifrom & Unifrom & Scattered & 2.14 & 0.7013 \\
\hline 43 & 46 & 70 & 0.9 & Feasible population & Adaptive feasible & Scattered & 2.236 & 0.6945 \\
\hline 19 & 47 & 80 & 0.7 & Feasible population & Unifrom & Scattered & 2.181 & 0.6985 \\
\hline 124 & 48 & 45 & 0.4 & Feasible population & Unifrom & Two point & 2.027 & 0.7115 \\
\hline 97 & 49 & 80 & 0.7 & Feasible population & Adaptive feasible & Single point & 2.282 & 0.691 \\
\hline 102 & 50 & 45 & 0.7 & Feasible population & Adaptive feasible & Single point & 2.276 & 0.6925 \\
\hline 23 & 51 & 45 & 0.7 & Feasible population & Unifrom & Scattered & 2.255 & 0.699 \\
\hline 64 & 52 & 45 & 0.7 & Unifrom & Unifrom & Single point & 2.169 & 0.691 \\
\hline 77 & 53 & 45 & 0.7 & Feasible population & Unifrom & Single point & 2.3965 & 0.709 \\
\hline 104 & 54 & 45 & 0.7 & Feasible population & Adaptive feasible & Single point & 2.2845 & 0.691 \\
\hline 138 & 55 & 45 & 1.0 & Unifrom & Adaptive feasible & Two point & 2.1815 & 0.722 \\
\hline 87 & 56 & 45 & 0.7 & Unifrom & Adaptive feasible & Single point & 2.283 & 0.691 \\
\hline 72 & 57 & 45 & 0.4 & Feasible population & Unifrom & Single point & 2.1245 & 0.7045 \\
\hline 11 & 58 & 45 & 0.7 & Unifrom & Unifrom & Scattered & 2.086 & 0.7095 \\
\hline 106 & 59 & 70 & 0.5 & Unifrom & Unifrom & Two point & 1.884 & 0.722 \\
\hline 143 & 60 & 45 & 0.7 & Unifrom & Adaptive feasible & Two point & 2.236 & 0.694 \\
\hline 110 & 61 & 80 & 0.7 & Unifrom & Unifrom & Two point & 1.987 & 0.7115 \\
\hline 25 & 62 & 45 & 0.7 & Feasible population & Unifrom & Scattered & 2.191 & 0.704 \\
\hline 151 & 63 & 45 & 1.0 & Feasible population & Adaptive feasible & Two point & 2.0955 & 0.7325 \\
\hline 53 & 64 & 20 & 0.5 & Unifrom & Unifrom & Single point & 2.095 & 0.708 \\
\hline 139 & 65 & 45 & 0.7 & Unifrom & Adaptive feasible & Two point & 2.189 & 0.698 \\
\hline 155 & 66 & 45 & 0.7 & Feasible population & Adaptive feasible & Two point & 2.148 & 0.6825 \\
\hline 68 & 67 & 20 & 0.9 & Feasible population & Unifrom & Single point & 2.0918 & 0.707 \\
\hline 60 & 68 & 45 & 1.0 & Unifrom & Unifrom & Single point & 2.1775 & 0.7045 \\
\hline 34 & 69 & 45 & 1.0 & Unifrom & Adaptive feasible & Scattered & 1.9043 & 0.7275 \\
\hline 74 & 70 & 45 & 0.7 & Feasible population & Unifrom & Single point & 2.137 & 0.703 \\
\hline 132 & 71 & 70 & 0.5 & Unifrom & Adaptive feasible & Two point & 2.285 & 0.691 \\
\hline 112 & 72 & 45 & 1.0 & Unifrom & Unifrom & Two point & 1.845 & 0.744 \\
\hline 3 & 73 & 20 & 0.9 & Unifrom & Unifrom & Scattered & 1.89 & 0.726 \\
\hline 4 & 74 & 70 & 0.9 & Unifrom & Unifrom & Scattered & 2.1 & 0.699 \\
\hline
\end{tabular}




\begin{tabular}{|c|c|c|c|c|c|c|c|c|}
\hline 75 & 75 & 45 & 0.7 & Feasible population & Unifrom & Single point & 2.0365 & 0.711 \\
\hline 46 & 76 & 45 & 0.4 & Feasible population & Adaptive feasible & Scattered & 2.285 & 0.691 \\
\hline 8 & 77 & 45 & 1.0 & Unifrom & Unifrom & Scattered & 2.0485 & 0.7115 \\
\hline 100 & 78 & 45 & 0.7 & Feasible population & Adaptive feasible & Single point & 2.2595 & 0.693 \\
\hline 76 & 79 & 45 & 0.7 & Feasible population & Unifrom & Single point & 2.1565 & 0.7055 \\
\hline 6 & 80 & 80 & 0.7 & Unifrom & Unifrom & Scattered & 1.956 & 0.699 \\
\hline 148 & 81 & 10 & 0.7 & Feasible population & Adaptive feasible & Two point & 2.116 & 0.703 \\
\hline 49 & 82 & 45 & 0.7 & Feasible population & Adaptive feasible & Scattered & 2.272 & 0.6915 \\
\hline 69 & 83 & 70 & 0.9 & Feasible population & Unifrom & Single point & 2.139 & 0.706 \\
\hline 24 & 84 & 45 & 0.7 & Feasible population & Unifrom & Scattered & 1.898 & 0.708 \\
\hline 150 & 85 & 45 & 0.4 & Feasible population & Adaptive feasible & Two point & 2.2825 & 0.6915 \\
\hline 126 & 86 & 45 & 0.7 & Feasible population & Unifrom & Two point & 2.265 & 0.7065 \\
\hline 42 & 87 & 20 & 0.9 & Feasible population & Adaptive feasible & Scattered & 2.218 & 0.6955 \\
\hline 79 & 88 & 20 & 0.5 & Unifrom & Adaptive feasible & Single point & 2.2325 & 0.6955 \\
\hline 80 & 89 & 70 & 0.5 & Unifrom & Adaptive feasible & Single point & 2.243 & 0.702 \\
\hline 18 & 90 & 10 & 0.7 & Feasible population & Unifrom & Scattered & 1.857 & 0.72 \\
\hline 105 & 91 & 20 & 0.5 & Unifrom & Unifrom & Two point & 1.998 & 0.729 \\
\hline 1 & 92 & 20 & 0.5 & Unifrom & Unifrom & Scattered & 1.9932 & 0.721 \\
\hline 10 & 93 & 45 & 0.7 & Unifrom & Unifrom & Scattered & 2.1545 & 0.701 \\
\hline 50 & 94 & 45 & 0.7 & Feasible population & Adaptive feasible & Scattered & 2.285 & 0.691 \\
\hline 153 & 95 & 45 & 0.7 & Feasible population & Adaptive feasible & Two point & 2.2655 & 0.6935 \\
\hline 71 & 96 & 80 & 0.7 & Feasible population & Unifrom & Single point & 2.1055 & 0.704 \\
\hline 85 & 97 & 45 & 0.4 & Unifrom & Adaptive feasible & Single point & 2.284 & 0.691 \\
\hline 92 & 98 & 20 & 0.5 & Feasible population & Adaptive feasible & Single point & 2.2065 & 0.698 \\
\hline 15 & 99 & 70 & 0.5 & Feasible population & Unifrom & Scattered & 1.857 & 0.7 \\
\hline 136 & 100 & 80 & 0.7 & Unifrom & Adaptive feasible & Two point & 2.2825 & 0.691 \\
\hline 133 & 101 & 20 & 0.9 & Unifrom & Adaptive feasible & Two point & 2.249 & 0.702 \\
\hline 67 & 102 & 70 & 0.5 & Feasible population & Unifrom & Single point & 2.115 & 0.7045 \\
\hline 57 & 103 & 10 & 0.7 & Unifrom & Unifrom & Single point & 1.56 & 0.7145 \\
\hline 29 & 104 & 20 & 0.9 & Unifrom & Adaptive feasible & Scattered & 2.216 & 0.7 \\
\hline 137 & 105 & 45 & 0.4 & Unifrom & Adaptive feasible & Two point & 2.279 & 0.6915 \\
\hline 142 & 106 & 45 & 0.7 & Unifrom & Adaptive feasible & Two point & 2.289 & 0.6965 \\
\hline 128 & 107 & 45 & 0.7 & Feasible population & Unifrom & Two point & 2.0955 & 0.721 \\
\hline 127 & 108 & 45 & 0.7 & Feasible population & Unifrom & Two point & 2.0955 & 0.721 \\
\hline 48 & 109 & 45 & 0.7 & Feasible population & Adaptive feasible & Scattered & 2.27 & 0.691 \\
\hline 12 & 110 & 45 & 0.7 & Unifrom & Unifrom & Scattered & 2.217 & 0.6965 \\
\hline 70 & 111 & 10 & 0.7 & Feasible population & Unifrom & Single point & 1.7225 & 0.756 \\
\hline 26 & 112 & 45 & 0.7 & Feasible population & Unifrom & Scattered & 2.178 & 0.699 \\
\hline 141 & 113 & 45 & 0.7 & Unifrom & Adaptive feasible & Two point & 2.2805 & 0.691 \\
\hline 35 & 114 & 45 & 0.7 & Unifrom & Adaptive feasible & Scattered & 1.867 & 0.7 \\
\hline 119 & 115 & 70 & 0.5 & Feasible population & Unifrom & Two point & 2.237 & 0.691 \\
\hline 96 & 116 & 10 & 0.7 & Feasible population & Adaptive feasible & Single point & 2.242 & 0.73 \\
\hline 22 & 117 & 45 & 0.7 & Feasible population & Unifrom & Scattered & 2.0633 & 0.723 \\
\hline 45 & 118 & 80 & 0.7 & Feasible population & Adaptive feasible & Scattered & 2.28 & 0.69115 \\
\hline 21 & 119 & 45 & 1.0 & Feasible population & Unifrom & Scattered & 2.007 & 0.717 \\
\hline 101 & 120 & 45 & 0.7 & Feasible population & Adaptive feasible & Single point & 2.285 & 0.6965 \\
\hline 54 & 121 & 70 & 0.5 & Unifrom & Unifrom & Single point & 1.915 & 0.709 \\
\hline 144 & 122 & 20 & 0.5 & Feasible population & Adaptive feasible & Two point & 2.1785 & 0.692 \\
\hline 154 & 123 & 45 & 0.7 & Feasible population & Adaptive feasible & Two point & 2.285 & 0.71 \\
\hline 120 & 124 & 20 & 0.9 & Feasible population & Unifrom & Two point & 1.999 & 0.72 \\
\hline 66 & 125 & 20 & 0.5 & Feasible population & Unifrom & Single point & 2.216 & 0.692 \\
\hline 125 & 126 & 45 & 1.0 & Feasible population & Unifrom & Two point & 1.8805 & 0.7355 \\
\hline 37 & 127 & 45 & 0.7 & Unifrom & Adaptive feasible & Scattered & 2.284 & 0.691 \\
\hline 107 & 128 & 20 & 0.9 & Unifrom & Unifrom & Two point & 1.6805 & 0.7485 \\
\hline 109 & 129 & 10 & 0.7 & Unifrom & Unifrom & Two point & 1.488 & 0.768 \\
\hline 61 & 130 & 45 & 0.7 & Unifrom & Unifrom & Single point & 1.981 & 0.72 \\
\hline 39 & 131 & 45 & 0.7 & Unifrom & Adaptive feasible & Scattered & 2.249 & 0.694 \\
\hline 117 & 132 & 45 & 0.7 & Unifrom & Unifrom & Two point & 2.0635 & 0.7115 \\
\hline 147 & 133 & 70 & 0.9 & Feasible population & Adaptive feasible & Two point & 1.8652 & 0.695 \\
\hline 94 & 134 & 20 & 0.9 & Feasible population & Adaptive feasible & Single point & 2.2625 & 0.6975 \\
\hline 41 & 135 & 70 & 0.5 & Feasible population & Adaptive feasible & Scattered & 2.2285 & 0.693 \\
\hline 145 & 136 & 70 & 0.5 & Feasible population & Adaptive feasible & Two point & 2.267 & 0.691 \\
\hline 36 & 137 & 45 & 0.7 & Unifrom & Adaptive feasible & Scattered & 2.284 & 0.692 \\
\hline 135 & 138 & 10 & 0.7 & Unifrom & Adaptive feasible & Two point & 1.7435 & 0.751 \\
\hline 40 & 139 & 20 & 0.5 & Feasible population & Adaptive feasible & Scattered & 2.28 & 0.692 \\
\hline 5 & 140 & 10 & 0.7 & Unifrom & Unifrom & Scattered & 1.914 & 0.736 \\
\hline 82 & 141 & 70 & 0.9 & Unifrom & Adaptive feasible & Single point & 2.266 & 0.6925 \\
\hline 7 & 142 & 45 & 0.4 & Unifrom & Unifrom & Scattered & 2.227 & 0.696 \\
\hline 56 & 143 & 70 & 0.9 & Unifrom & Unifrom & Single point & 2.1145 & 0.7085 \\
\hline 123 & 144 & 80 & 0.7 & Feasible population & Unifrom & Two point & 2.1085 & 0.7055 \\
\hline 81 & 145 & 20 & 0.9 & Unifrom & Adaptive feasible & Single point & 2.2105 & 0.6935 \\
\hline 146 & 146 & 20 & 0.9 & Feasible population & Adaptive feasible & Two point & 2.1445 & 0.7105 \\
\hline 113 & 147 & 45 & 0.7 & Unifrom & Unifrom & Two point & 1.9215 & 0.7525 \\
\hline 116 & 148 & 45 & 0.7 & Unifrom & Unifrom & Two point & 1.9215 & 0.696 \\
\hline 152 & 149 & 45 & 0.7 & Feasible population & Adaptive feasible & Two point & 2.206 & 0.6935 \\
\hline 52 & 150 & 45 & 0.7 & Feasible population & Adaptive feasible & Scattered & 2.2605 & 0.713 \\
\hline 129 & 151 & 45 & 0.7 & Feasible population & Unifrom & Two point & 2.0065 & 0.713 \\
\hline 99 & 152 & 45 & 1.0 & Feasible population & Adaptive feasible & Single point & 2.004 & 0.713 \\
\hline 16 & 153 & 20 & 0.9 & Feasible population & Unifrom & Scattered & 2.107 & 0.731 \\
\hline 55 & 154 & 20 & 0.9 & Unifrom & Unifrom & Single point & 1.783 & 0.7265 \\
\hline 20 & 155 & 45 & 0.4 & Feasible population & Unifrom & Scattered & 2.114 & 0.706 \\
\hline 33 & 156 & 45 & 0.4 & Unifrom & Adaptive feasible & Scattered & 2.2095 & 0.696 \\
\hline
\end{tabular}

A. ANOVA for the Response Surface Quadratic Model

The nature of the model for the MRR and TWR found to be quadratic. Therefore, the optimum conditions have been predicated efficiently by the predicted model. F-test and probability test has been performed in order to check the significance of the models. F-ratio is defined as the ratio between groups means square values to within group. Table 4 implies the model is significant having F-value of 7.04. There is only a $0.01 \%$ chance that a "Model F-Value" this large could occur due to noise.

Table 4: ANOVA for Response Surface Quadratic Model for MRR

\begin{tabular}{|l|c|c|c|c|c|c|}
\hline Source & Sum of & DF & Mean & F Value & Prob $>\mathrm{F}$ & \\
\hline
\end{tabular}




\begin{tabular}{|c|c|c|c|c|c|c|}
\hline & Squares & & Square & & & \\
\hline Model & 2.45 & 22 & 0.11 & 7.04 & $<0.0001$ & significant \\
\hline A & 0.50 & 1 & 0.50 & 31.65 & $<0.0001$ & \\
\hline $\mathrm{B}$ & 0.11 & 1 & 0.11 & 7.02 & 0.0090 & \\
\hline $\mathrm{C}$ & 0.057 & 1 & 0.057 & 3.59 & 0.0605 & \\
\hline $\mathrm{D}$ & 1.05 & 1 & 1.05 & 66.28 & $<0.0001$ & \\
\hline $\mathrm{E}$ & 0.11 & 2 & 0.054 & 3.42 & 0.0355 & \\
\hline$A^{2}$ & 0.40 & 1 & 0.40 & 25.10 & $<0.0001$ & \\
\hline $\mathrm{B}^{2}$ & $4.907 \times 10^{-3}$ & 1 & $4.907 \times 10^{-3}$ & 0.31 & 0.5784 & \\
\hline $\mathrm{AB}$ & $9.750 \times 10^{-3}$ & 1 & $9.750 \times 10^{-3}$ & 0.62 & 0.4338 & \\
\hline $\mathrm{AC}$ & 0.072 & 1 & 0.072 & 4.53 & 0.0351 & \\
\hline $\mathrm{AD}$ & 0.022 & 1 & 0.022 & 1.36 & 0.2449 & \\
\hline $\mathrm{AE}$ & 0.052 & 2 & 0.026 & 1.65 & 0.1954 & \\
\hline $\mathrm{BC}$ & 0.012 & 1 & 0.012 & 0.75 & 0.3878 & \\
\hline $\mathrm{BD}$ & $2.976 \times 10^{-3}$ & 1 & $2.976 \times 10^{-3}$ & 0.19 & 0.6652 & \\
\hline $\mathrm{BE}$ & $5.644 \times 10^{-3}$ & 2 & $2.822 \times 10^{-3}$ & 0.18 & 0.8368 & \\
\hline $\mathrm{CD}$ & $5.858 \times 10^{-4}$ & 1 & $5.858 \times 10^{-3}$ & 0.037 & 0.8477 & \\
\hline $\mathrm{CE}$ & 0.013 & 2 & $6.279 \times 10^{-3}$ & 0.40 & 0.6731 & \\
\hline $\mathrm{DE}$ & 0.040 & 2 & 0.020 & 1.26 & 0.2882 & \\
\hline Residual & 2.10 & 133 & 0.016 & & & \\
\hline Lack of Fit & 1.54 & 85 & 0.018 & 1.54 & 0.0511 & not significant \\
\hline Pure Error & 0.56 & 48 & 0.012 & & & \\
\hline Cor Total & 4.55 & 155 & & & & \\
\hline
\end{tabular}

\section{B. Optimum GA Parameters Predicted by RSM}

To find the optimum Genetic Algorithm parameters by RSM (i.e. numerical, graphical etc.) various techniques and methods have been used. By applying numerical optimization of Design expert 6.0 version with the use of RSM, as shown in Table 5, optimum parameters have been predicted. The optimum GA parameters for the maximization of MRR are - population size $: 55$, Crossover : 0.5, creation function: Feasible population, mutation: adaptive feasible ,crossover function: Two point have been predicted in the MATLAB environment. The Model F-value of 8.42 implies the model is significant. There is only a $0.01 \%$ chance that a "Model F-Value" this large could occur due to noise.

Table 5: Anova for Response Surface Quadratic Model for TWR

\begin{tabular}{|c|c|c|c|c|c|c|}
\hline Source & Sum of Squares & DF & Mean Square & F Value & Prob>F & \\
\hline ModeL & 0.023 & 22 & $1.027 \times 10^{-3}$ & 8.42 & $<0.0001$ & significant \\
\hline A & $6.216 \times 10^{-3}$ & 1 & $6.216 \times 10^{-3}$ & 50.96 & $<0.0001$ & \\
\hline $\mathrm{B}$ & $2.359 \times 10^{-3}$ & 1 & $2.35 \times 10^{-3}$ & 19.34 & $<0.0001$ & \\
\hline $\mathrm{C}$ & $8.13 \times 10^{-5}$ & 1 & $8.135 \times 10^{-5}$ & 0.67 & 0.4156 & \\
\hline $\mathrm{D}$ & $8.079 \times 10^{-3}$ & 1 & $8.079 \times 10^{-3}$ & 66.24 & $<0.0001$ & \\
\hline $\mathrm{E}$ & $1.854 \times 10^{-3}$ & 2 & $9.272 \times 10^{-4}$ & 7.60 & 0.0007 & \\
\hline $\mathrm{A}^{2}$ & $1.801 \times 10^{-3}$ & 1 & $1.801 \times 10^{-3}$ & 14.76 & 0.0002 & \\
\hline $\mathrm{B}^{2}$ & $1.640 \times 10^{-4}$ & 1 & $1.640 \times 10^{-4}$ & 1.34 & 0.2482 & \\
\hline $\mathrm{AB}$ & $2.034 \times 10^{-4}$ & 1 & $2.034 \times 10^{-4}$ & 1.67 & 0.1989 & \\
\hline $\mathrm{AC}$ & $1.231 \times 10^{-4}$ & 1 & $1.231 \times 10^{-4}$ & 1.01 & 0.3168 & \\
\hline $\mathrm{AD}$ & $2.896 \times 10^{-4}$ & 1 & $2.896 \times 10^{-4}$ & 2.37 & 0.1257 & \\
\hline $\mathrm{AE}$ & $3.334 \times 10^{-4}$ & 2 & $1.667 \times 10^{-4}$ & 1.37 & 0.2585 & \\
\hline $\mathrm{BC}$ & $1.606 \times 10^{-6}$ & 1 & $1.606 \times 10^{-6}$ & 0.013 & 0.9088 & \\
\hline $\mathrm{BD}$ & $1.256 \times 10^{-5}$ & 1 & $1.256 \times 10^{-5}$ & 0.10 & 0.7488 & \\
\hline $\mathrm{BE}$ & $3.588 \times 10^{-4}$ & 2 & $1.794 \times 10^{-4}$ & 1.47 & 0.2335 & \\
\hline $\mathrm{CD}$ & $2.31 \times 10^{-5}$ & 1 & $2.31 \times 10^{-5}$ & 0.19 & 0.6640 & \\
\hline $\mathrm{CE}$ & $2.439 \times 10^{-4}$ & 2 & $1.220 \times 10^{-4}$ & 1.00 & 0.3706 & \\
\hline $\mathrm{DE}$ & $5.502 \times 10^{-4}$ & 2 & $2.751 \times 10^{-4}$ & 2.26 & 0.1088 & \\
\hline Residual & 0.016 & 133 & $1.220 \times 10^{-4}$ & & & \\
\hline Lack of Fit & 0.012 & 85 & $1.389 \times 10^{-4}$ & 1.51 & 0.0602 & not significant \\
\hline Pure Error & $4.412 \mathrm{E}-003$ & 48 & $9.192 \times 10^{-5}$ & & & \\
\hline
\end{tabular}


Genetic Algorithm Parameters Optimization For Electrical Discharge Machining Using Design Of

Experiments

\begin{tabular}{|l|l|l|l|l|l|l|}
\hline Cor Total & 0.039 & 155 & & & \\
\hline
\end{tabular}

Table 6: Criteria for Optimization

\begin{tabular}{|l|l|l|l|l|}
\hline Name & Goal & Lower Limit & Upper Limit & Importance \\
\hline Population size & is in range & 20 & 70 & 3 \\
\hline Crossover fraction & is in range & 0.5 & 0.9 & 3 \\
\hline Creation function & is in range & Unifrom & Feasible population & 3 \\
\hline Mutation & is in range & Unifrom & Adaptive feasible & 3 \\
\hline Crossover function & is in range & Scattered & Two point & 3 \\
\hline MRR & Maximize & 1.488 & 2.3965 & 3 \\
\hline TWR & Minimize & 0.67 & 0.768 & 3 \\
\hline
\end{tabular}

Table 7: Optimum Parameter Selection

\begin{tabular}{|c|c|c|c|c|c|c|c|c|}
\hline No. & $\begin{array}{c}\text { Population } \\
\text { size }\end{array}$ & $\begin{array}{c}\text { Crossover } \\
\text { Fraction }\end{array}$ & $\begin{array}{c}\text { Creation } \\
\text { function }\end{array}$ & Mutation & $\begin{array}{c}\text { Crossover } \\
\text { function }\end{array}$ & $\begin{array}{c}\text { MRR } \\
{[\mathbf{m g} / \mathbf{s}]}\end{array}$ & $\begin{array}{c}\text { TWR } \\
{[\mathbf{m g} / \mathbf{s}]}\end{array}$ & Desirability \\
\hline 1 & 55 & 0.5 & $\mathrm{~A}$ & $\mathrm{~B}$ & $\mathrm{C}$ & 2.28625 & 0.685199 & 0.862 \\
\hline 2 & 64 & 0.5 & $\mathrm{D}$ & $\mathrm{B}$ & $\mathrm{C}$ & 2.3181 & 0.689386 & 0.856 \\
\hline 3 & 50 & 0.5 & $\mathrm{~A}$ & $\mathrm{~B}$ & $\mathrm{C}$ & 2.31755 & 0.691021 & 0.847 \\
\hline 4 & 45 & 0.5 & $\mathrm{~A}$ & $\mathrm{~B}$ & $\mathrm{E}$ & 2.29071 & 0.688822 & 0.845 \\
\hline 5 & 53 & 0.5 & $\mathrm{D}$ & $\mathrm{B}$ & $\mathrm{E}$ & 2.2903 & 0.688805 & 0.845 \\
\hline 6 & 51 & 0.5 & $\mathrm{D}$ & $\mathrm{B}$ & $\mathrm{E}$ & 2029037 & 0.688932 & 0.844 \\
\hline
\end{tabular}

Feasible population (A), Adaptive feasible (B), Two point (C), Unifron (D),Scattered (E)

\section{CONCLUSION}

The present work considers the GA parameters optimization for MRR and TWR in the Electric Discharge Machining (EDM). A Central composite design (CCD) of response surface model (RSM) with 5 levels has been considered for the optimization. Total of 156 experiments have been performed in the genetic algorithm by varying its different parameters. From the different experiments conducted by Genetic Algorithm in the MATLAB environment, the optimum values for maximize MRR and minimize TWR at population size 55,crossover 0.5,creation function:Feasible population, mutation: adaptive feasible ,crossover function: Two point have been obtained.

\section{REFERENCES}

[1] Tzeng CY and Chen RY, " Optimization of Electric Discharge Machining Process Using the Response Surface Methodology and Genetic Algorithm Approach International Journal of precision engineering and manufacturing”, vol 14,no 5, Journal of Zhejiang University SCIENCE AISSN 1673-565X (Print); ISSN 1862-1775 (Online)www.zju.edu.cn/jzus;www.springerlink.comE-mail: jzus@zju.edu.cn, pp. 709-717

[2] Yadav SK. and Azad D, “Ann modeling and multi objective optimization of edm process", ELK Asia Pacific Journals - Special Issue isbn: 978-81930411-4-7 ann ,pp. 112-117.

[3] Radhika N, Shivaram P, Karthik KT, "Multi-Objective Optimization in Electric Discharge Machining

of Aluminium Composite", Vol. 36, No. 4 (2014) 428-436 Tribology in Industry www.tribology.fink.rs ,pp. 428-436

[4] Qing G and Zhang.Q, “ Parameter optimization model in electrical discharge machining process”, Journal of Zhejiang University SCIENCE A ISSN 1673-565X (Print); ISSN 1862-1775 (Online) www.zju.edu.cn/jzus; www.springerlink.com E-mail: jzus@ @ju.edu.cn,pp.104-108.

[5] Balasubramaniana P and Senthilvelan T, "Optimization of Machining Parameters in EDM process using Cast and Sintered Copper Electrodes", 22118128 (C) 2014 Elsevier Ltd. This is an open access article under the CC BY-NC-ND license (http://creativecommons.org/licenses/by-nc-nd/3.0/). Selection and peer review under responsibility of the Gokaraju Rangaraju Institute of Engineering and Technology (GRIET) doi: 10.1016/j.mspro.2014.07.108,pp.1292 - 1302 .

[6] Tiwari RK , "Multi-objective optimization of electrical discharge machining process parameters using Genetic algorithm", International Journal of Engineering Research and General Science Volume 3, Issue 3, May-June, 2015 ISSN 2091-2730,pp. 1411-1423

[7] Ahmad M ,Davis R, Maurya. N, Singh P \& Gupta S, "Optimzation of process parameters in electric discharge machining process", International Journal of Mechanical Engineering (IJME) ISSN(P): 2319-2240;ISSN(E) 2319-2259 Vol. 5, Issue 4, Jun - Jul 2016; pp.45-52 .

[8] Harmanpreet and Harvinder L , "Investigation and modeling and of edm process parameters in machining of incoloy-800", IJRET: International Journal of Research in Engineering and Technology eISSN: 2319-1163 | pISSN: 2321-7308 Volume: 04 Issue: 08 | August-2015, Available @ http://www.ijret.org,pp. 112-117.

[9] Laxma J and Raj K G, "Mathematical modeling and analysis of EDM process parameters based onTaguchi design of experiments", International Conference on Vibration Problems (ICOVP-2015) IOP Publishing Journal of Physics: Conference Series 662 (2015) 012025 doi:10.1088/17426596/662/1/012025 pp. 1-10.

[10] Periyakgounder S, Venkatesan R, Sekar T and Elango N, "Optimization of Intervening Variables in MicroEDM of SS 316L using a Genetic Algorithm and Response-SurfaceMethodology”, Strojniški vestnik - Journal of Mechanical Engineering 60(2014)10, 656-664 Received for review: 2014-01-10@ 2014 Journal of Mechanical Engineering. All rights reserved. Received revised form: 2014-0520 DOI:10.5545/sv-jme.2014.1665,pp. 656-664. 